\title{
Bariatric Surgery versus Intensive Medical Therapy for Diabetes - 3-Year Outcomes
}

\author{
Philip R. Schauer, M.D., Deepak L. Bhatt, M.D., M.P.H., John P. Kirwan, Ph.D., Kathy Wolski, \\ M.P.H., Stacy A. Brethauer, M.D., Sankar D. Navaneethan, M.D., M.P.H., Ali Aminian, M.D., \\ Claire E. Pothier, M.P.H., Esther S.H. Kim, M.D., M.P.H., Steven E. Nissen, M.D., Sangeeta R. \\ Kashyap, M.D., and for the STAMPEDE Investigators ${ }^{*}$ \\ Bariatric and Metabolic Institute (P.R.S., S.A.B., A.A.), Lerner Research Institute (J.P.K.), Heart \\ and Vascular Institute (K.W., C.E.P., E.S.H.K., S.E.N.), Urological and Kidney Institute (S.D.N.), \\ and Endocrinology Institute (S.R.K.), Cleveland Clinic, Cleveland; and Brigham and Women's \\ Hospital Heart and Vascular Center and Harvard Medical School — both in Boston (D.L.B.)
}

\section{Abstract}

BACKGROUND—In short-term randomized trials (duration, 1 to 2 years), bariatric surgery has been associated with improvement in type 2 diabetes mellitus.

\begin{abstract}
METHODS-We assessed outcomes 3 years after the randomization of 150 obese patients with uncontrolled type 2 diabetes to receive either intensive medical therapy alone or intensive medical therapy plus Roux-en-Y gastric bypass or sleeve gastrectomy. The primary end point was a glycated hemoglobin level of $6.0 \%$ or less.
\end{abstract}

RESULTS-The mean $( \pm \mathrm{SD})$ age of the patients at baseline was $48 \pm 8$ years, $68 \%$ were women, the mean baseline glycated hemoglobin level was $9.3 \pm 1.5 \%$, and the mean baseline body-mass index (the weight in kilograms divided by the square of the height in meters) was $36.0 \pm 3.5$. A total of $91 \%$ of the patients completed 36 months of follow-up. At 3 years, the criterion for the primary end point was met by $5 \%$ of the patients in the medical-therapy group, as compared with $38 \%$ of those in the gastric-bypass group $(\mathrm{P}<0.001)$ and $24 \%$ of those in the sleeve-gastrectomy group ( $\mathrm{P}$ $=0.01)$. The use of glucose-lowering medications, including insulin, was lower in the surgical groups than in the medical-therapy group. Patients in the surgical groups had greater mean percentage reductions in weight from baseline, with reductions of $24.5 \pm 9.1 \%$ in the gastric-bypass group and $21.1 \pm 8.9 \%$ in the sleeve-gastrectomy group, as compared with a reduction of $4.2 \pm 8.3 \%$ in the medical-therapy group ( $\mathrm{P}<0.001$ for both comparisons). Quality-of-life measures were significantly better in the two surgical groups than in the medical-therapy group. There were no major late surgical complications.

CONCLUSIONS-Among obese patients with uncontrolled type 2 diabetes, 3 years of intensive medical therapy plus bariatric surgery resulted in glycemic control in significantly more patients

Address reprint requests to Dr. Schauer at the Bariatric and Metabolic Institute, Cleveland Clinic, M61, 9500 Euclid Ave., Cleveland, OH 44195, or at schauep@ccf.org.

The contributions of the authors and committee members in the Surgical Treatment and Medications Potentially Eradicate Diabetes Efficiently (STAMPEDE) trial are listed in the Supplementary Appendix, available at NEJM.org.

No other potential conflict of interest relevant to this article was reported.

Disclosure forms provided by the authors are available with the full text of this article at NEJM.org. 
than did medical therapy alone. Analyses of secondary end points, including body weight, use of glucose-lowering medications, and quality of life, also showed favorable results at 3 years in the surgical groups, as compared with the group receiving medical therapy alone. (Funded by Ethicon and others; STAMPEDE ClinicalTrials.gov number, NCT00432809.)

Bariatric surgery has recently emerged as a potentially useful treatment for type 2 diabetes mellitus. ${ }^{1}$ Observational studies ${ }^{2-5}$ and randomized, controlled trials ${ }^{6-10}$ have shown that procedures including Roux-en-Y gastric bypass, sleeve gastrectomy, gastric banding, and biliopancreatic diversion significantly improve glycemic control and favorably affect cardiovascular risk factors.

In the Surgical Treatment and Medications Potentially Eradicate Diabetes Efficiently (STAMPEDE) trial, we found that 1 year after randomization, gastric bypass and sleeve gastrectomy were superior to intensive medical therapy alone in achieving glycemic control and reducing cardiovascular risk factors while decreasing dependency on pharmacotherapy for diabetes management. ${ }^{7}$ Although bariatric surgery yields short-term improvements in glycemic control, questions remain regarding the durability of the metabolic benefits of surgery, long-term safety, quality of life, and effects on diabetes-related end-organ disease. The current report provides results of the 3-year follow-up analyses from the STAMPEDE trial and addresses other unanswered questions about the durability of the benefits of bariatric surgery as compared with intensive medical therapy for treating diabetes mellitus.

\section{METHODS}

\section{STUDY DESIGN}

The rationale, design, and methods of the study have been reported previously. ${ }^{7,11}$ The complete protocol was approved by the institutional review board at the Cleveland Clinic and is available with the full text of this article at NEJM.org. Briefly, the trial was a threegroup, randomized, controlled, single-center study involving 150 obese patients, in which the effects of intensive medical therapy were compared with those of gastric bypass or sleeve gastrectomy. With the use of block randomization, patients were assigned, in a 1:1:1 ratio, to one of the three study groups, with stratification according to the baseline use of insulin. Eligibility criteria included an age of 20 to 60 years, a glycated hemoglobin level of more than $7.0 \%$, and a body-mass index (BMI, the weight in kilograms divided by the square of the height in meters) of 27 to 43 . All patients provided written informed consent.

\section{STUDY OUTCOMES}

The primary outcome was a glycated hemoglobin level of $6.0 \%$ or less, with or without the use of diabetes medications. ${ }^{7,11}$ This report provides 3 -year outcomes in the study patients, including measures of glycemic control, weight loss, blood pressure, lipid levels, renal function, carotid intima-media thickness, ${ }^{12}$ medication use, adverse events, disease-related complications, and quality of life (as evaluated with the use of the RAND 36-Item Health Survey). ${ }^{13}$ The strategy for all three groups was the adjustment of medical therapy (every 3 months for 2 years and every 6 months thereafter) with the goal of achieving the therapeutic target of a glycated hemoglobin level of $6.0 \%$ or less, without unacceptable side effects associated with medical treatment. 


\section{STUDY OVERSIGHT}

This investigator-initiated trial was financially supported by Ethicon, with additional support from LifeScan, the Cleveland Clinic, and the National Institutes of Health. The sponsors participated in discussions regarding study design but had no role in data accrual, data analysis, or manuscript preparation. The first author wrote the first draft of the manuscript. All the authors had full and independent access to all the data and vouch for the integrity and the accuracy of the analysis and its fidelity to the protocol. Complete study governance is outlined in the Supplementary Appendix, available at NEJM.org.

\section{STATISTICAL ANALYSIS}

We report all continuous variables with a normal distribution as means and standard deviations. Variables with a non-normal distribution are reported as medians and interquartile ranges. Categorical variables are summarized with the use of frequencies. We used the chi-square test to evaluate the primary end point of a glycated hemoglobin level of $6.0 \%$ or less at 3 years. We used an analysis of variance to analyze continuous laboratory measurements and to perform comparisons among the three study groups. For glycemic measures and body weight, a mixed model for repeated measures was used to analyze the change from baseline, and least-square means with corresponding standard errors were plotted graphically.

A stepwise multivariable logistic model was used to determine factors associated with achieving the primary end point. Factors that were considered in the model included age, sex, insulin use, duration of diabetes, baseline glycated hemoglobin and glucose levels, Cpeptide levels, baseline BMI, change in BMI, blood pressure, and lipid measures. No adjustments were made for multiple comparisons, since these were exploratory analyses. Analyses were performed with the use of SAS software, version 9.2 (SAS Institute).

\section{RESULTS}

\section{STUDY PATIENTS}

Of the 150 patients who underwent randomization from March 2007 through January 2011, a total of 9 patients withdrew from the trial immediately after randomization or during the initial 6 months ( 8 patients in the medical-therapy group and 1 patient who did not undergo sleeve gastrectomy because of severe anemia); 4 patients were lost to follow-up. The remaining 137 patients $(91.3 \%$ ) were evaluated in the 3-year assessment of safety and efficacy.

The baseline characteristics of the 150 patients were reported previously. ${ }^{7}$ In the current analysis, $68 \%$ of the patients were women, and $74 \%$ were white. The mean $( \pm \mathrm{SD})$ age was $48 \pm 8$ years, and the mean BMI was $36 \pm 3.5 ; 49$ patients (36\%) had a BMI of less than 35 . The mean glycated hemoglobin level was $9.3 \pm 1.5 \%$, and the average duration of diabetes was $8.3 \pm 5.1$ years, with $43 \%$ of patients requiring insulin at baseline. There were no significant differences between the study groups at baseline (Table S1 in the Supplementary Appendix). 


\section{PRIMARY END POINT}

At 3 years, the target glycated hemoglobin level of $6.0 \%$ or less was achieved in $5 \%$ of the patients in the medical-therapy group, as compared with $38 \%$ of those in the gastric-bypass group $(\mathrm{P}<0.001)$ and $24 \%$ of those in the sleeve-gastrectomy group $(\mathrm{P}=0.01)$ (Table 1$)$. The percentage of patients who had a glycated hemoglobin level of $6.0 \%$ or less at 1 year but did not maintain this level of glycemic control at 3 years (which was defined as a glycemic relapse) was $80 \%$ in the medical-therapy group, as compared with $24 \%$ in the gastric-bypass group $(\mathrm{P}=0.03)$ and $50 \%$ in the sleeve-gastrectomy group $(\mathrm{P}=0.34)$. In the entire cohort, a reduction in the BMI was the only significant predictor of achieving the primary end point (odds ratio for each 1-unit decrease in BMI, 1.41; 95\% confidence interval [CI], 1.22 to 1.64; $\mathrm{P}<0.001$ ) (Table $\mathrm{S} 2$ in the Supplementary Appendix). In the two surgical groups, meeting the criterion for the primary end point was predicted both by a reduction in the BMI (odds ratio, $1.33 ; 95 \% \mathrm{CI}, 1.15$ to $1.56 ; \mathrm{P}<0.001$ ) and by a duration of diabetes of less than 8 years (odds ratio, $3.3 ; 95 \% \mathrm{CI}, 1.2$ to $9.1 ; \mathrm{P}=0.02$ ).

\section{GLYCEMIC CONTROL}

After 3 years, each of the two surgical procedures was superior to intensive medical therapy alone in achieving exploratory targets for glycated hemoglobin of $6.5 \%$ and $7.0 \%$, with or without the use of diabetes medications ( $\mathrm{P}<0.05$ for all comparisons) (Table 1). Median levels of fasting plasma glucose were significantly lower in the two surgical groups than in the medical-therapy group ( $\mathrm{P}<0.01$ for both comparisons) (Table 1$)$. There were more rapid, larger, and more sustained reductions in levels of glycated hemoglobin and fasting plasma glucose and in the use of glucose-lowering medications in the two surgical groups than in the medical-therapy group (Table 2, Fig. 1A and 1C, and Fig. S1 in the Supplementary Appendix). The reductions in glycated hemoglobin levels, medication use, and BMI in the surgical groups were similar in patients with a BMI of less than 35 and those with a BMI of 35 or more, and the reductions in both BMI subgroups of the surgical group were greater than the reductions in either BMI subgroup of the medical-therapy group (Fig. 1B, and Fig. S2 and S3 in the Supplementary Appendix).

\section{DIABETES MEDICATIONS}

At 3 years, the use of glucose-lowering medications including insulin was reduced from baseline in the two surgical groups (Table 2). Patients in the gastric-bypass group required fewer glucose-lowering medications per day than did those in the sleeve-gastrectomy group $(0.48 \pm 0.80$ vs. $1.02 \pm 1.01)$. The proportion of patients who were not taking any glucoselowering medications was significantly higher in the gastric-bypass group than in the sleevegastrectomy group (Table 2).

\section{WEIGHT LOSS}

At 3 years, reductions in body weight, BMI, waist circumference, and waist-to-hip ratio were greater after gastric bypass and sleeve gastrectomy than after intensive medical therapy (Table 1, Fig. 1D, and Table S3 in the Supplementary Appendix). The reduction in body weight was greater after gastric bypass than after sleeve gastrectomy $(\mathrm{P}=0.02)$. 


\section{CARDIOVASCULAR BIOMARKERS AND MEDICATIONS}

The decrease in triglyceride levels and increase in high-density lipoprotein (HDL) cholesterol levels that had been observed after the two surgical procedures, as compared with intensive medical therapy, were sustained at 3 years (Table 1). There were no significant differences in blood pressure and low-density lipoprotein (LDL) cholesterol levels among the three study groups, although there was a significant reduction in the number of medications needed to treat hyperlipidemia and hypertension in the surgical groups (Table S4 in the Supplementary Appendix). There were no significant differences among the three groups in maximal carotid intima-media thickness at baseline or at 24 months (Table S5 in the Supplementary Appendix).

\section{RENAL OUTCOMES}

At 3 years, the urinary albumin-to-creatinine ratio (as measured in milligrams of albumin to grams of creatinine) decreased from a median of 9 to 6 in the gastric-bypass group $(\mathrm{P}=$ $0.08)$ and from 12 to 7 in the sleeve-gastrectomy group $(\mathrm{P}<0.001)$, as compared with 6.5 to 5.5 in the medical-therapy group $(\mathrm{P}=0.77)$. The reduction in the albumin-to-creatinine ratio from baseline in the two surgery groups was significant, as compared with the medicaltherapy group ( $\mathrm{P}<0.04$ for both comparisons) (Table S5 in the Supplementary Appendix). Of the patients with baseline albuminuria, a return to normal values at 3 years occurred in 8 of 13 patients $(62 \%)$ in the gastric-bypass group ( $\mathrm{P}=0.04$ for the within-group comparison), 8 of 10 patients $(80 \%)$ in the sleeve-gastrectomy group $(\mathrm{P}=0.11)$, and 1 of 4 patients $(25 \%)$ in the medical-therapy group $(\mathrm{P}=1.00)$. There was no significant difference in the serum creatinine level and the estimated glomerular filtration rate among the three groups during follow-up (Table S5 in the Supplementary Appendix).

\section{QUALITY OF LIFE}

There were significant improvements in five of eight mental and physical domains among patients in the gastric-bypass group and in two of eight domains among patients in the sleeve-gastrectomy group, as compared with the medical-therapy group (Fig. 2, and Table S6 in the Supplementary Appendix).

\section{ADVERSE EVENTS}

Additional surgical interventions were required in 4 patients within the first 12 months after randomization, ${ }^{7}$ but none were performed thereafter. There was no excessive weight loss or hypoalbuminemia and no life-threatening complications or deaths in any of the groups (Table 3, and Tables S7 and S8 in the Supplementary Appendix). Excessive weight gain ( $>5 \%$ from baseline) was observed in 7 of 43 patients $(16 \%)$ in the medical-therapy group and in no patients in the two surgical groups $(\mathrm{P}<0.05$ for both comparisons).

\section{DISCUSSION}

The results of this follow-up analysis show that 3 years after randomization, bariatric surgery, as compared with intensive medical therapy alone, was associated with superior and sustained glycemic control and weight reduction. Patients who underwent gastric bypass or sleeve gastrectomy were significantly more likely to achieve and maintain a glycated 
hemoglobin level of $6.0 \%$ or less than were those who received intensive medical therapy alone. More than one third of the patients in the gastric-bypass group and a fifth of those in the sleeve-gastrectomy group, as compared with no patients in the medical-therapy group, had a glycated hemoglobin level of $6.0 \%$ or less without the use of diabetes medications. Patients in the two surgical groups had a significant absolute decrease of 2.5 percentage points in glycated hemoglobin levels, a reduction that was sustained for 3 years, as compared with a reduction of 0.6 percentage points in the medical-therapy group. The results of surgery are particularly striking in this population with relatively long-standing uncontrolled disease. The surgically treated patients had superior glycemic control for 3 years while also reducing dependency on oral diabetes medications and insulin. More than $90 \%$ of surgical patients had glycemic control without the use of insulin. Weight loss and a shorter duration of diabetes were the main predictors of having a glycated hemoglobin level of $6.0 \%$ or less after surgery.

Analysis of secondary end points, including BMI, body weight, waist circumference, and levels of triglycerides and HDL cholesterol, also showed favorable results at 3 years in the surgical groups, as compared with the group receiving intensive medical therapy alone. Patients in the two surgical groups had a significant reduction in the use of antihypertensive and lipid-lowering agents, even though there were no significant changes from baseline measurements in blood pressure or LDL cholesterol among the three study groups. Some adverse effects of surgical treatment were observed in this study but were modest in severity and relatively uncommon after the first year.

Although observational studies have shown impressive improvements in glycemic control after bariatric surgery, with rates of improvement in diabetes ranging from 55 to $95 \%,{ }^{1-5}$ direct comparisons with intensive medical therapy have been limited. ${ }^{6-10}$ The Swedish Obese Subjects (SOS) study, a nonrandomized, prospective trial comparing bariatric surgery with conventional medical treatment, showed higher rates of diabetes remission after surgery than after conventional medical treatment at 2, 10, and 20 years. The SOS study also showed significant reductions in long-term complications, including rates of death from any cause and major cardiovascular events, with surgical treatment. ${ }^{14-16}$

Five short-term, randomized, controlled trials compared bariatric surgery with medical treatment with respect to type 2 diabetes, with 1 to 2 years of follow-up. ${ }^{6-10}$ The initial STAMPEDE report showed that at 1 year, patients had better glycemic control (defined as a glycated hemoglobin level of $6.0 \%$ or less, with or without the use of medications) after gastric bypass ( $42 \%$ of patients) or sleeve gastrectomy (37\%) than after intensive medical therapy $(12 \%)\left(\mathrm{P}<0.001\right.$ for both comparisons). ${ }^{7}$ All five trials showed that standard bariatric procedures, as compared with medical treatment alone, were associated with few major complications and resulted in superior glycemic control, weight reduction, and reductions in cardiovascular risk factors. Our findings show continued durability of glycemic control and persistent reductions in cardiovascular risk factors at 3 years after surgery. The diabetes remission rates after surgery in our study are similar to those reported by Ikramuddin et al. ${ }^{9}$ but are lower than those reported by Mingrone et al. ${ }^{8}$ Such discrepancies could be explained by the greater severity and longer duration of diabetes in our population, as well as a stricter definition of remission. 
Observational studies have suggested that bariatric surgery may reduce long-term renal impairment associated with diabetes. ${ }^{4,17}$ Diabetes and obesity are independent risk factors for the development of albuminuria, which is associated with an increased risk of death from cardiovascular causes and end-stage renal disease. ${ }^{18}$ We noted improvement in albuminuria in the surgical groups despite a reduction in the use of renin-angiotensin system blockers, which suggests that bariatric surgery may have a role in the prevention of further renal parenchymal damage. Although there was no significant change in serum creatinine levels or the glomerular filtration rate, these findings do not imply a lack of benefit of weight loss on renal function, since creatinine and the glomerular filtration rate are influenced by loss of muscle mass associated with weight loss. ${ }^{19}$ Cumulatively, our data should be considered to be hypothesis-generating and suggest the need for further long-term studies examining the effects of bariatric surgery on renal function in diabetes.

Obese patients with diabetes have a reduced quality of life and ability to cope with associated chronic diseases. Using a validated quality-of-life instrument, we found significant improvements in five of eight mental and physical domains among patients in the gastric-bypass group and in two of eight domains among patients in the sleeve-gastrectomy group. Intensive medical therapy resulted in no significant improvements from baseline in quality of life.

Metabolic and weight-loss outcomes were generally similar in the two surgical groups at 1 year, although some advantages of gastric bypass over sleeve gastrectomy have emerged during longer follow-up, including a greater likelihood of reaching a glycated hemoglobin level of 7.0\% or less (a therapeutic goal of the American Diabetes Association) with no use of diabetes medications, a reduced requirement for diabetes and cardiovascular medications, greater reductions in weight and BMI, and a greater improvement in quality of life. Some differences between the gastric-bypass group and the sleeve-gastrectomy group did not reach statistical significance, although the study was not adequately powered to detect modest differences between these procedures. In a prespecified substudy analysis of beta-cell function, insulin sensitivity, and body composition in a subgroup of patients, we found that at 2 years, gastric bypass was superior to sleeve gastrectomy with respect to insulin secretion, insulin sensitivity, and relative reduction in truncal fat as compared with subcutaneous fat. ${ }^{20}$

Most clinical guidelines and insurance coverage for bariatric surgery limit access to the surgery to patients with a BMI of 35 or more, presumably because of insufficient studies evaluating outcomes in patients with a BMI of less than 35. In our study, 49 of 137 patients (36\%) had a BMI of 27 to 34 , and these patients had an improvement in glycemic control and durability that was similar to that in patients with a BMI of 35 or more. Other trials and observational studies involving patients with only mild obesity showed similar improvements in glycemic control. ${ }^{21,22}$ Accordingly, some guidelines for diabetes management are beginning to endorse the use of bariatric surgery in patients with diabetes and a BMI of 30 to 34 , especially those who have poor glycemic control despite receiving the best available medical therapy. ${ }^{23,24}$ 
Important limitations of our study include an inadequate sample size and duration to detect differences in the incidence of diabetes complications, such as myocardial infarction, stroke, or death. The protocol specifies further follow-up at 5 years for all patients, which should allow additional assessment of even longer-term efficacy and safety.

Despite these limitations, we conclude that bariatric surgery represents a potentially useful strategy for the management of type 2 diabetes, allowing many patients to reach and maintain therapeutic targets of glycemic control that otherwise would not be achievable with intensive medical therapy alone. Some patients in our study had complete diabetes remission, whereas others had a marked reduction in the need for pharmacologic treatment. The reduction in cardiovascular risk factors was sustained, allowing for reductions in lipidlowering and antihypertensive therapies. Other benefits of surgery included a significant improvement in the quality of life. The question as to whether the documented benefits will reduce microvascular and macrovascular morbidity and mortality, as shown in nonrandomized studies, can be adequately answered only through larg0065r, multicenter clinical-outcome trials.

\section{Supplementary Material}

Refer to Web version on PubMed Central for supplementary material.

\section{Acknowledgments}

Supported by grants from Ethicon (EES IIS 19900), the Investigator-Initiated Study Program of LifeScan, the Cleveland Clinic, and the National Institutes of Health (R01 DK089547).

Dr. Schauer reports receiving consulting fees from Ethicon and Novo Nordisk, lecture fees from Ethicon, Eli Lilly, Nestle, and Global Academy for Medical Education, fees for board membership and stock options or equity from Surgiquest, Barosense, Remedy MD, and SE Healthcare Quality Consulting, fees for providing expert testimony from Physicians Review of Surgery regarding complications of surgery, and financial support for a fellowship program from Stryker and having a pending patent application with the Cleveland Clinic regarding medical devices for weight loss; Dr. Bhatt, receiving consulting fees from Medscape Cardiology, receiving honoraria for serving on a steering committee from WebMD, having a pending consulting relationship with Regado Biosciences, and receiving grant support from Amarin, AstraZeneca, Bristol-Myers Squibb, Eisai, Ethicon, Medtronic, SanofiAventis, the Medicines Company, FlowCo, PLx Pharma, Roche, and Takeda; Dr. Kirwan, receiving grant support from Nestle, lecture fees from Eli Lilly, Novo Nordisk, and International Life Sciences Institute, and honoraria from Cereal Partners Worldwide; Dr. Kim, receiving grant support from GE and consulting fees from Philips; Dr. Nissen, receiving grant support through his institution from AstraZeneca, Anthera, Amgen, Karo Bio, Pfizer, the Medicines Company, Novartis, Takeda, Resverlogix, Ethicon, Orexigen, Vivus, and Eli Lilly; and Dr. Kashyap, receiving consulting fees from Ethicon.

We thank Chytaine Hall for patient-retention support; Josephine Chan, Ph.D., Craig Balog, Debbie Gladish, Andrew Pikus, and Randy Scott for statistics and data-management support; Matthew Kroh, M.D., Tomasz Rogula, M.D., Bipan Chand, M.D., Derick Cetin, D.O., Archana Gorty, M.D., Bartolome Burguera, M.D., Ph.D., Betul Hatipoglu, M.D., Mario Skugor, M.D., Adi Mehta, M.D., Leslie Heinberg, Ph.D., Julie Merrell, Ph.D., Kathleen Ashton, Ph.D., Megan Lavery, Ph.D., Ellen Calogeras, Wendy Kirby, and Lauren Sullivan for medical-site support; Rishi Singh, M.D., Lisa Yerian, M.D., Eva Balazs, Karilane King, Erin Mayock, Roman Poliszczuk for technical support; and Suzanne Turner and Mary Ann Citraro for graphical support.

\section{REFERENCES}

1. Vest AR, Heneghan HM, Agarwal S, Schauer PR, Young JB. Bariatric surgery and cardiovascular outcomes: a systematic review. Heart. 2012; 98:1763-1777. [PubMed: 23077152] 
2. Pories WJ, Swanson MS, MacDonald KG, et al. Who would have thought it? An operation proves to be the most effective therapy for adult-onset diabetes mellitus. Ann Surg. 1995; 222:339-350. [PubMed: 7677463]

3. Schauer PR, Burguera B, Ikramuddin S, et al. Effect of laparoscopic Roux-en Y gastric bypass on type 2 diabetes mellitus. Ann Surg. 2003; 238:467-484. [PubMed: 14530719]

4. Brethauer SA, Aminian A, Romero-Talamás H, et al. Can diabetes be surgically cured? Long-term metabolic effects of bariatric surgery in obese patients with type 2 diabetes mellitus. Ann Surg. 2013; 258:628-636. [PubMed: 24018646]

5. Adams TD, Davidson LE, Litwin SE, et al. Health benefits of gastric bypass surgery after 6 years. JAMA. 2012; 308:1122-1131. [PubMed: 22990271]

6. Dixon JB, O'Brien PE, Playfair J, et al. Adjustable gastric banding and conventional therapy for type 2 diabetes: a randomized controlled trial. JAMA. 2008; 299:316-323. [PubMed: 18212316]

7. Schauer PR, Kashyap SR, Wolski K, et al. Bariatric surgery versus intensive medical therapy in obese patients with diabetes. N Engl J Med. 2012; 366:1567-1576. [PubMed: 22449319]

8. Mingrone G, Panunzi S, De Gaetano A, et al. Bariatric surgery versus conventional medical therapy for type 2 diabetes. N Engl J Med. 2012; 366:1577-1585. [PubMed: 22449317]

9. Ikramuddin S, Korner J, Lee WJ, et al. Roux-en-Y gastric bypass vs intensive medical management for the control of type 2 diabetes, hypertension, and hyperlipidemia: the Diabetes Surgery Study randomized clinical trial. JAMA. 2013; 309:2240-2249. [PubMed: 23736733]

10. Liang Z, Wu Q, Chen B, Yu P, Zhao H, Ouyang X. Effect of laparoscopic Rouxen-Y gastric bypass surgery on type 2 diabetes mellitus with hypertension: a randomized controlled trial. Diabetes Res Clin Pract. 2013 May 22. (Epub ahead of print).

11. Kashyap SR, Bhatt DL, Schauer PR. Bariatric surgery vs0 advanced practice medical management in the treatment of type 2 diabetes mellitus: rationale and design of the Surgical Therapy And Medications Potentially Eradicate Diabetes Efficiently trial (STAMPEDE). Diabetes Obes Metab. 2010; 12:452-454. [Erratum, Diabetes Obes Metab 2010;12:833.]. [PubMed: 20415694]

12. Stein JH, Korcarz CE, Hurst RT, et al. Use of carotid ultrasound to identify subclinical vascular disease and evaluate cardiovascular disease risk: a consensus statement from the American Society of Echocardiography Carotid Intima-Media Thickness Task Force. J Am Soc Echocardiogr. 2008; 21:93-111. [Erratum, J Am Soc Echocardiogr 2008;21:376.]. [PubMed: 18261694]

13. Hays RD, Sherbourne CD, Mazel RM. The RAND 36-Item Health Survey 1.0. Health Econ. 1993; 2:217-227. [PubMed: 8275167]

14. Sjöström L, Lindroos AK, Peltonen M, et al. Lifestyle, diabetes, and cardiovascular risk factors 10 years after bariatric surgery. N Engl J Med. 2004; 351:2683-2693. [PubMed: 15616203]

15. Sjöström L, Narbro K, Sjöström CD, et al. Effects of bariatric surgery on mortality in Swedish obese subjects. N Engl J Med. 2007; 357:741-752. [PubMed: 17715408]

16. Sjöström L, Peltonen M, Jacobson P, et al. Bariatric surgery and long-term cardiovascular events. JAMA. 2012; 307:56-65. [PubMed: 22215166]

17. Iaconelli A, Panunzi S, De Gaetano A, et al. Effects of bilio-pancreatic diversion on diabetic complications: a 10-year follow-up. Diabetes Care. 2011; 34:561-567. [PubMed: 21282343]

18. Chronic Kidney Disease Prognosis Consortium. Association of estimated glomerular filtration rate and albuminuria with all-cause and cardiovascular mortality in general population cohorts: a collaborative meta-analysis. Lancet. 2010; 375:2073-2081. [PubMed: 20483451]

19. Jesudason DR, Clifton P. Interpreting different measures of glomerular filtration rate in obesity and weight loss: pitfalls for the clinician. Int J Obes (Lond). 2012; 36:1421-1427. [PubMed: 22184061]

20. Kashyap SR, Bhatt DL, Wolski K, et al. Metabolic effects of bariatric surgery in patients with moderate obesity and type 2 diabetes: analysis of a randomized control trial comparing surgery with intensive medical treatment. Diabetes Care. 2013; 36:2175-2182. [PubMed: 23439632]

21. Maggard-Gibbons M, Maglione M, Livhits M, et al. Bariatric surgery for weight loss and glycemic control in nonmorbidly obese adults with diabetes: a systematic review. JAMA. 2013; 309:2250 2261. [PubMed: 23736734] 
22. Cohen RV, Pinheiro JC, Schiavon CA, Salles JE, Wajchenberg BL, Cummings DE. Effects of gastric bypass surgery in patients with type 2 diabetes and only mild obesity. Diabetes Care. 2012; 35:1420-1428. [PubMed: 22723580]

23. Dixon JB, Zimmet P, Alberti KG, Rubino F. Bariatric surgery: an IDF statement for obese type 2 diabetes. Diabet Med. 2011; 28:628-642. [PubMed: 21480973]

24. Mechanick JI, Youdim A, Jones DB, et al. Clinical practice guidelines for the perioperative nutritional, metabolic, and nonsurgical support of the bariatric surgery patient - 2013 update: cosponsored by American Association of Clinical Endocrinologists, the Obesity Society, and American Society for Metabolic \& Bariatric Surgery. Surg Obes Relat Dis. 2013; 9:159-191. [PubMed: 23537696] 


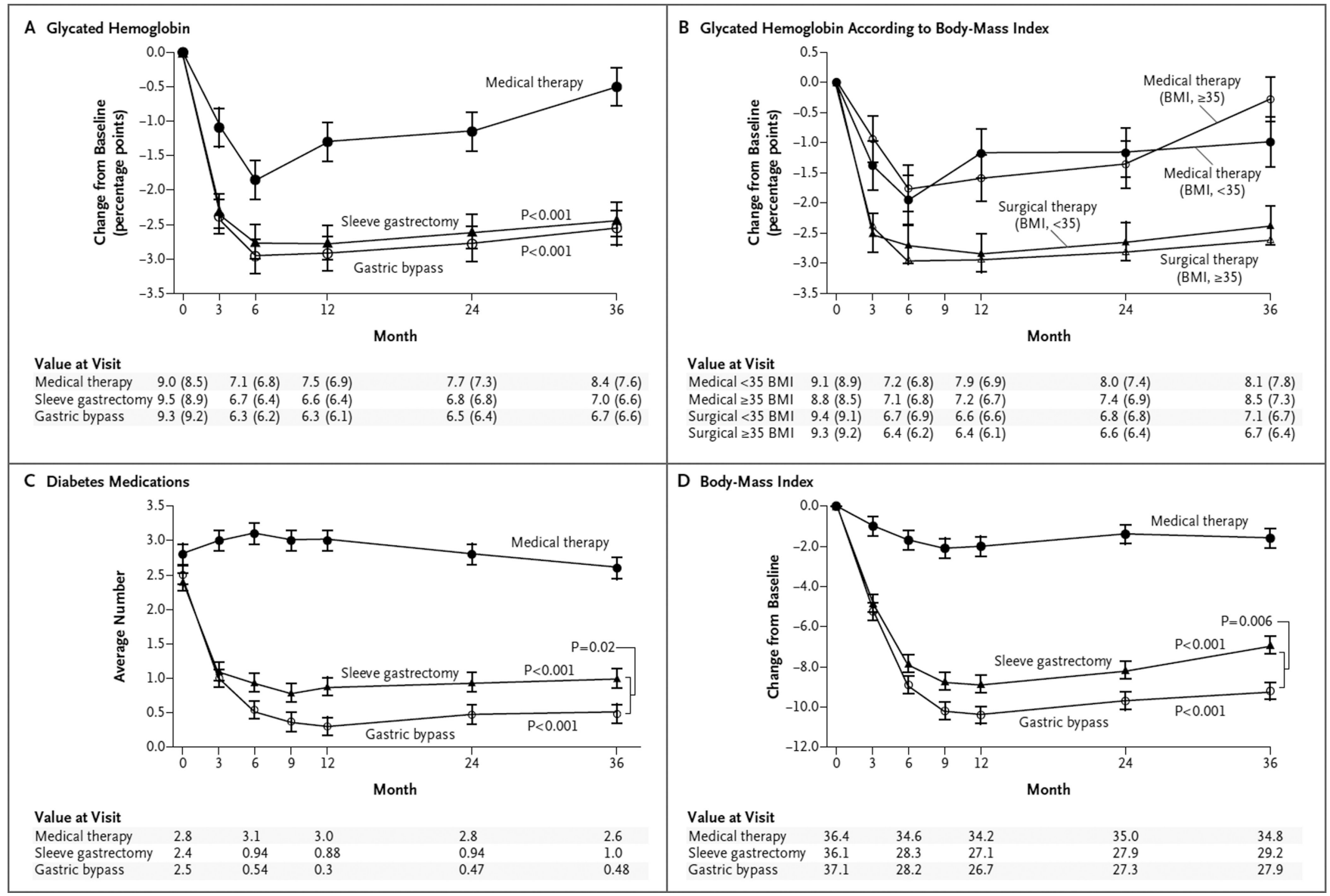

Figure 1. Mean Changes in Measures of Diabetes Control from Baseline to 3 Years

Shown are the percentage change in glycated hemoglobin levels (Panel A), the percentage change in glycated hemoglobin levels according to body-mass index (BMI) (Panel B), the average number of diabetes medications during the study period (Panel $\mathrm{C}$ ), and the changes in BMI (Panel D) over a 3-year period among patients receiving intensive medical therapy only, sleeve gastrectomy, or gastric bypass. I bars indicate standard errors. Mean values in each group are provided below the graphs; in Panels A and B, median values are also provided in parentheses. $\mathrm{P}$ values are for the comparison between each surgical group and the medical-therapy group in Panels A, C, and D. In Panel B, P $=0.008$ for the comparison between the surgical groups and the medical-therapy group for the subgroup of patients with a BMI of less than 35; $\mathrm{P}<0.001$ for the comparison for the subgroup with a BMI of 35 or more. 


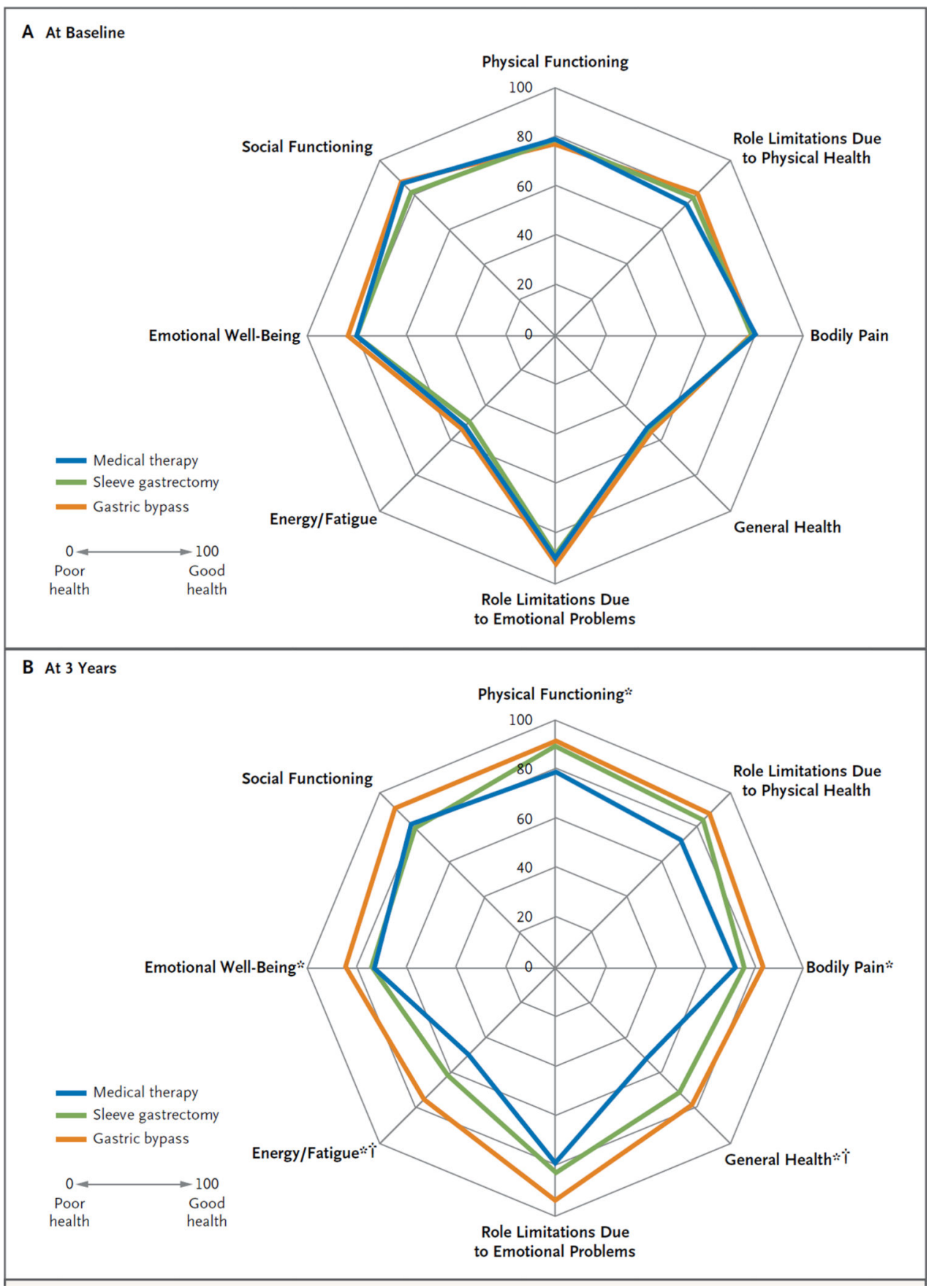

Figure 2. Polar Chart of Scores for Quality of Life at Baseline and 3 Years after Randomization The scores on the RAND 36-Item Health Survey range from the worst score of 0 (poor health) to the best score of 100 (good health). Asterisks indicate $\mathrm{P}<0.05$ for the comparison between the gastric-bypass group and the medical-therapy group; daggers indicate $\mathrm{P}<0.05$ for the comparison between the sleeve-gastrectomy group and the medical-therapy group. The minimally important difference (MID) in scoring for this survey is unknown. 


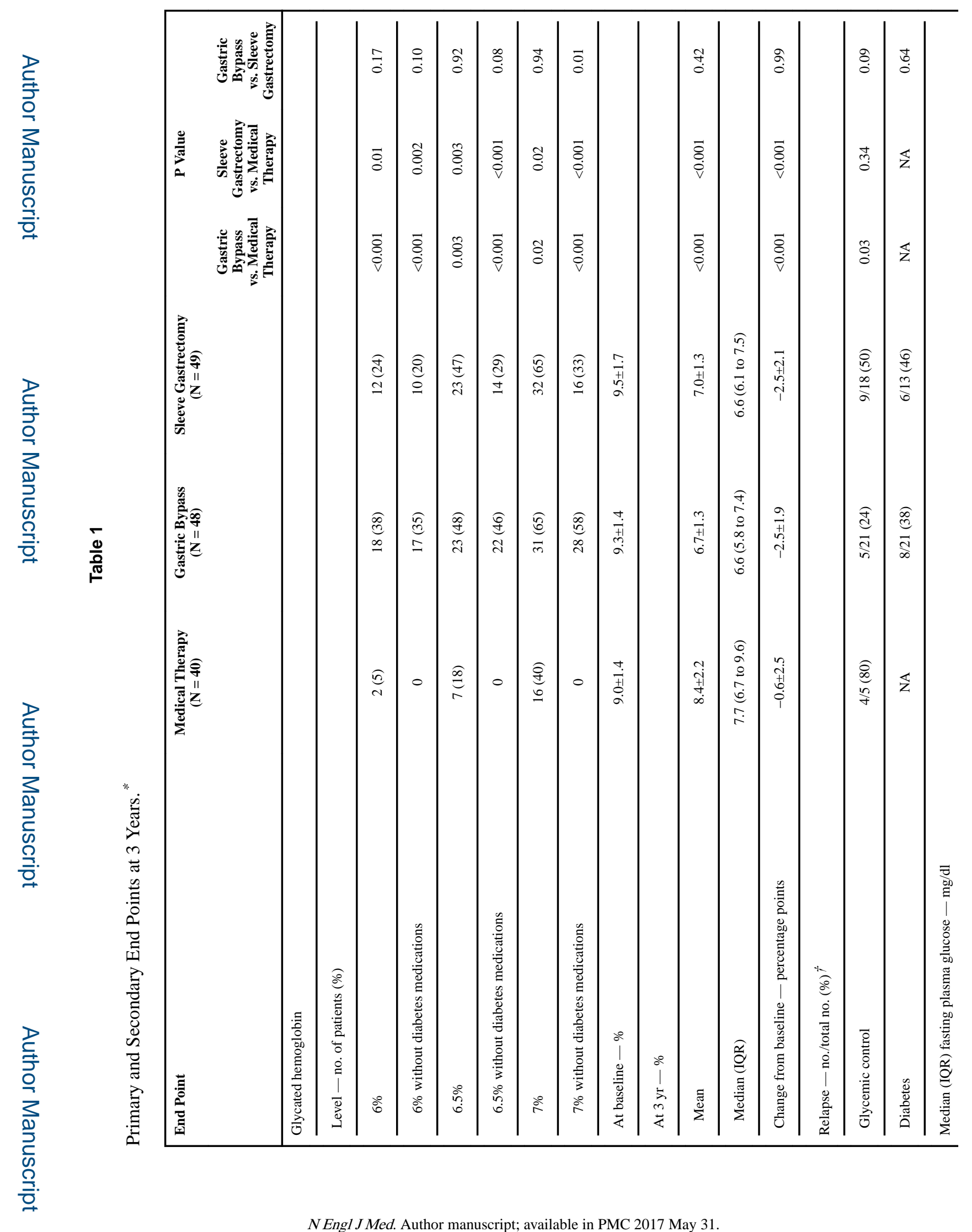




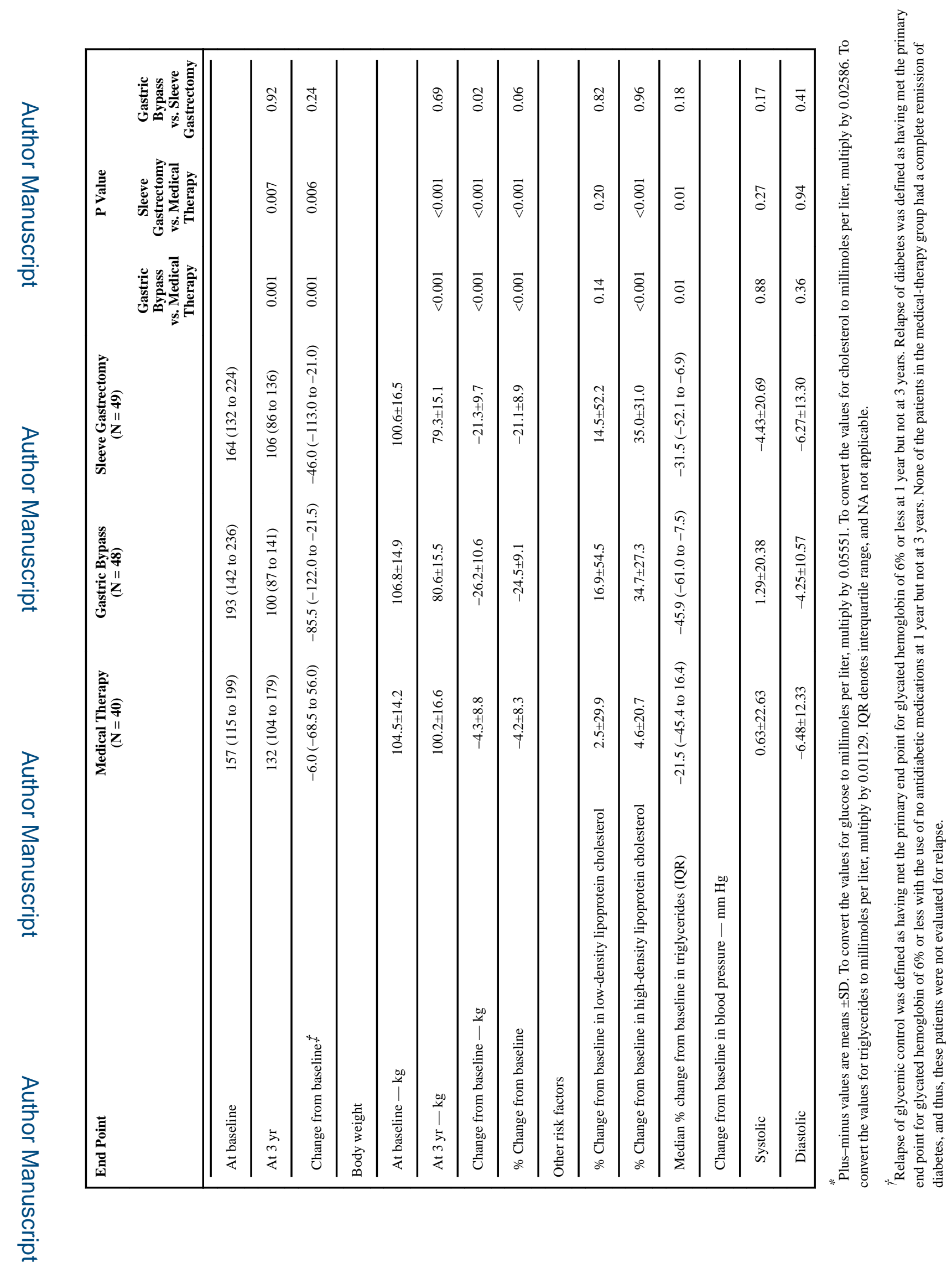

N Engl J Med. Author manuscript; available in PMC 2017 May 31. 


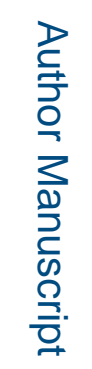

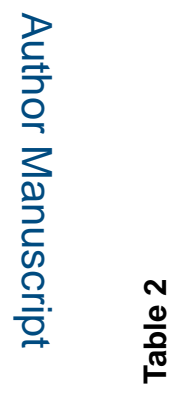

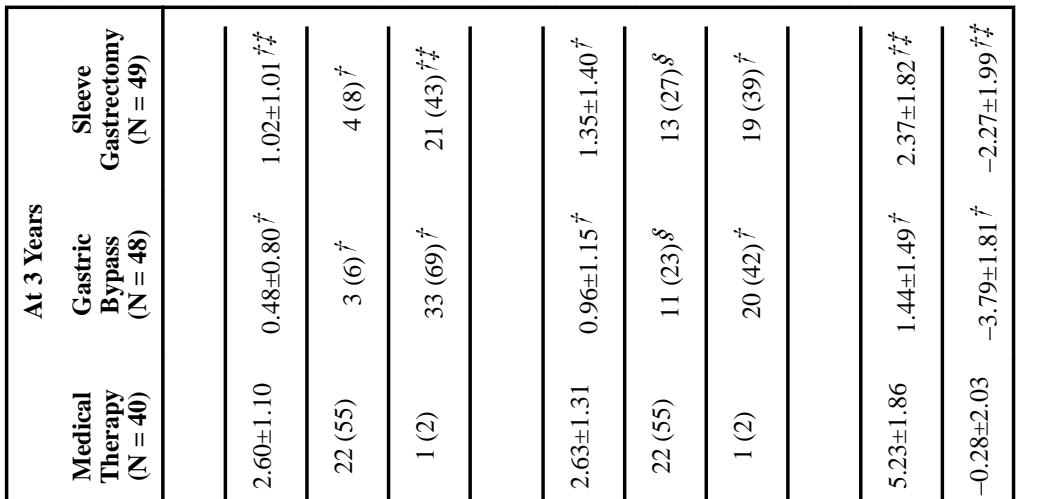

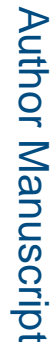
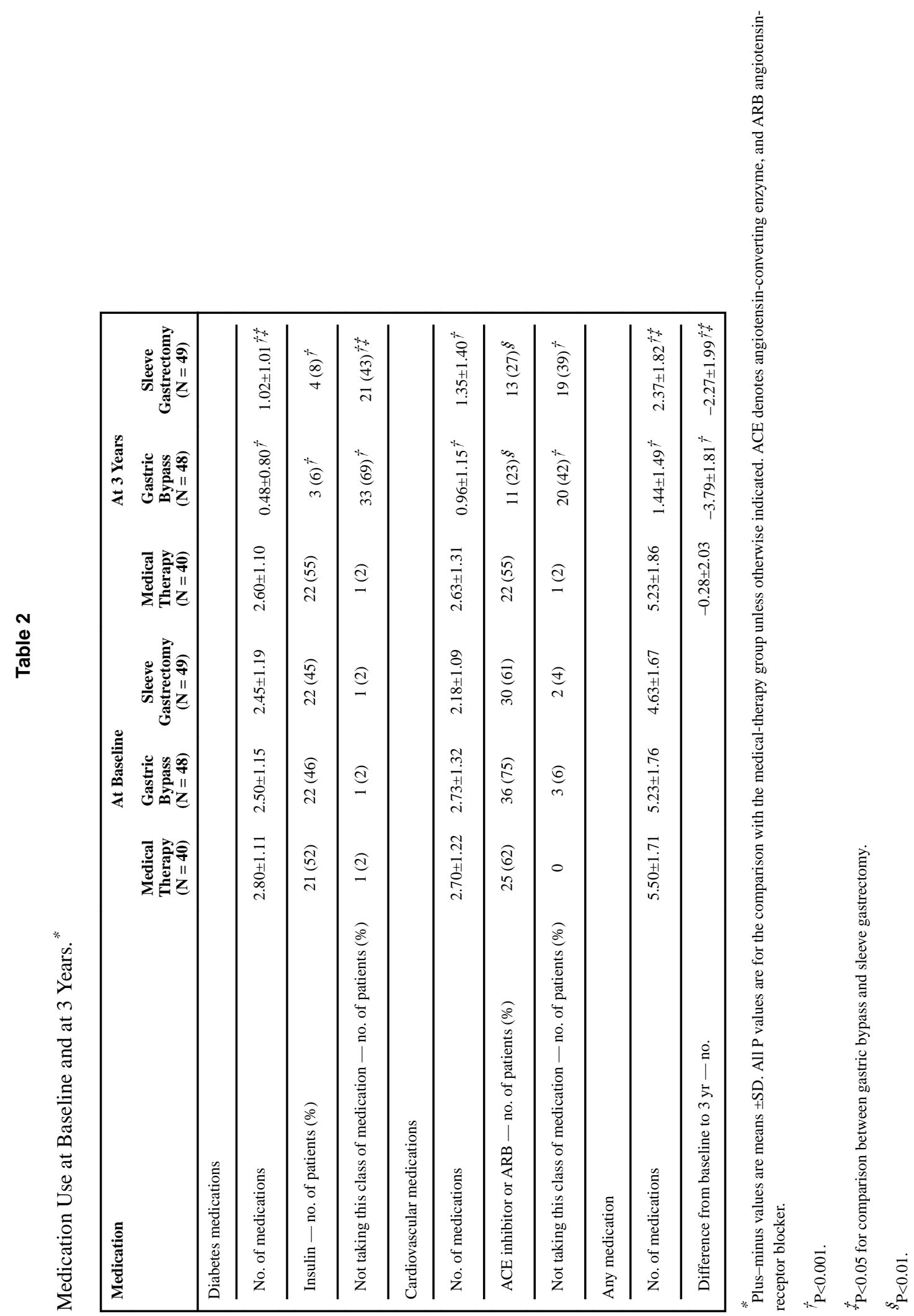

NEngl J Med. Author manuscript; available in PMC 2017 May 31. 
Table 3

Complications at 3 Years.*

\begin{tabular}{|c|c|c|c|}
\hline Complication & $\begin{array}{l}\text { Medical Therapy } \\
(\mathrm{N}=43)\end{array}$ & $\begin{array}{l}\text { Gastric Bypass } \\
(\mathbf{N}=50) \\
\text { number of patients (percent) }\end{array}$ & $\begin{array}{l}\text { Sleeve Gastrectomy } \\
(\mathbf{N}=49)\end{array}$ \\
\hline \multicolumn{4}{|l|}{ Gastrointestinal } \\
\hline Bowel obstruction & $1(2)$ & $1(2)$ & $1(2)$ \\
\hline Stricture & 0 & $1(2)$ & $1(2)$ \\
\hline Ulcer & $1(2)$ & $4(8)$ & 0 \\
\hline Leak & 0 & 0 & $1(2)$ \\
\hline Intraabdominal bleeding & 0 & $2(4)$ & 0 \\
\hline Dumping syndrome & 0 & $4(8)$ & $1(2)$ \\
\hline Gallstone diseases & 0 & $1(2)$ & $1(2)$ \\
\hline \multicolumn{4}{|l|}{ Microvascular or macrovascular } \\
\hline Stroke & 0 & 0 & $1(2)$ \\
\hline Retinopathy & 0 & $1(2)$ & $2(4)$ \\
\hline Nephropathy ${ }^{\dagger}$ & $4(9)$ & $7(14)$ & $5(10)$ \\
\hline Foot ulcer & 0 & $2(4)$ & $1(2)$ \\
\hline \multicolumn{4}{|l|}{ Nutritional and metabolic } \\
\hline Anemia & $6(14)$ & $8(16)$ & $15(31)$ \\
\hline Intravenous treatment for dehydration & $3(7)$ & $7(14)$ & $4(8)$ \\
\hline Hypoglycemic episode & $39(91)$ & $32(64)$ & $40(82)$ \\
\hline Severe hypoglycemia requiring intervention & 0 & $1(2)$ & 0 \\
\hline Excessive weight gain $t$ & $7(16)$ & 0 & 0 \\
\hline \multicolumn{4}{|l|}{ Other } \\
\hline Wound infection & 0 & $1(2)$ & 0 \\
\hline Hernia & $1(2)$ & $3(6)$ & $1(2)$ \\
\hline Pneumonia & 0 & $2(4)$ & $1(2)$ \\
\hline Renal calculus & $6(14)$ & $5(10)$ & $4(8)$ \\
\hline Cancer & $2(5)$ & $2(4)$ & $2(4)$ \\
\hline
\end{tabular}

* Not included in the safety analysis were seven patients in the medical-therapy group who withdrew immediately after randomization and one patient in the sleeve-gastrectomy group who had anemia before withdrawing from the study before surgery. Five patients who started the study but later withdrew or were lost to follow-up are included in this analysis until their discontinuation.

${ }^{\dagger}$ Nephropathy was defined as any one of the following criteria: doubling of the serum creatinine level or a decrease in the glomerular filtration rate of more than 20\%; development of macroalbuminuria (urine albumin-to-creatinine ratio, $>300$ [as measured in milligrams of albumin to grams of creatinine]); or renal transplantation, initiation of dialysis, or an increase in the serum creatinine level of more than $3.3 \mathrm{mg}$ per deciliter $(290 \mu \mathrm{mol}$ per liter) in the absence of an acute reversible cause.

${ }^{t}$ Excessive weight gain was defined as a $5 \%$ increase in body weight over baseline. $\mathrm{P}<0.05$ for the comparison between the medical-therapy group and each of the surgical groups. 